\title{
Pattern Selection in Growing Tubular Tissues
}

\author{
P. Ciarletta, ${ }^{1,2}$ V. Balbi, ${ }^{1}$ and E. Kuhl ${ }^{3, *}$ \\ ${ }^{1}$ CNRS and Sorbonne Universités, Université Paris 6, Institut Jean le Rond d'Alembert, UMR 7190, \\ 4 Place Jussieu case 162, 75005 Paris, France \\ ${ }^{2}$ MOX and Fondazione CEN, Politecnico di Milano, Piazza Leonardo da Vinci 32, 20133 Milano, Italy \\ ${ }^{3}$ Living Matter Laboratory, Stanford University, 496 Lomita Mall, Stanford, California 94305, USA
}

(Received 4 August 2014; published 9 December 2014)

\begin{abstract}
Tubular organs display a wide variety of surface morphologies including circumferential and longitudinal folds, square and hexagonal undulations, and finger-type protrusions. Surface morphology is closely correlated to tissue function and serves as a clinical indicator for physiological and pathological conditions, but the regulators of surface morphology remain poorly understood. Here, we explore the role of geometry and elasticity on the formation of surface patterns. We establish morphological phase diagrams for patterns selection and show that increasing the thickness or stiffness ratio between the outer and inner tubular layers induces a gradual transition from circumferential to longitudinal folding. Our results suggest that physical forces act as regulators during organogenesis and give rise to the characteristic circular folds in the esophagus, the longitudinal folds in the valves of Kerckring, the surface networks in villi, and the crypts in the large intestine.
\end{abstract}

DOI: 10.1103/PhysRevLett.113.248101

PACS numbers: 87.17.Pq, 46.32.+x, 87.18.Hf

Tubular tissues are found ubiquitously in living systems constituting the fundamental building blocks of many organs. Characterized by a multilayered organization with a highly functionalized microstructural architecture, they perform a wide range of tasks ranging from gas exchange in airways to blood transport in arteries. In vertebrates, histological differences in tissue anatomy arise during early embryonic development after a series of processes, which are shared across many species [1]. For example, in the gastrointestinal tract, the anteroposterior division of the tubular structure occurs after gastrulation from the differentiation of two layers: the endoderm, which gives rise to the epithelium, and the mesoderm, which later develops into the smooth muscle layer, the mesenchyme [2]. To functionalize the digestive activities of the gastrointestinal tract, various epithelial structures will later emerge, such as circular folds in the esophagus, longitudinal folds in the valves of Kerckring in the jejunum, and surface networks of villi or crypts in the large intestine [3] (see Fig. 1, top). Epigenetic factors are of paramount importance throughout organogenesis, as endoderm-mesoderm interactions have been found to direct the formation of various surface patterns that characterize the adult epithelia [4]. During the first stage of morphogenesis, different rates of cell proliferation in the two layers give rise to residual stresses to promote a mechanical instability, which triggers the occurrence of regional specificities. This buckling effect has recently been observed in embryonic intestinal tissues, where the spatial confinement caused by muscle differentiation has been proven to drive early epithelial morphologies in different species [5]. During the second stage, physical forces at the interface activate signaling pathways at the cellular level and interact with individual morphogens and transcription factors to determine the expression of a specific form of cell differentiation during later stages of organogenesis [6].

Elastic effects are not only important to initiate epithelial differentiation in the early embryo - they also drive morphological transitions in adult tubular tissues, which often indicate the onset of a disease. In fact, pathological

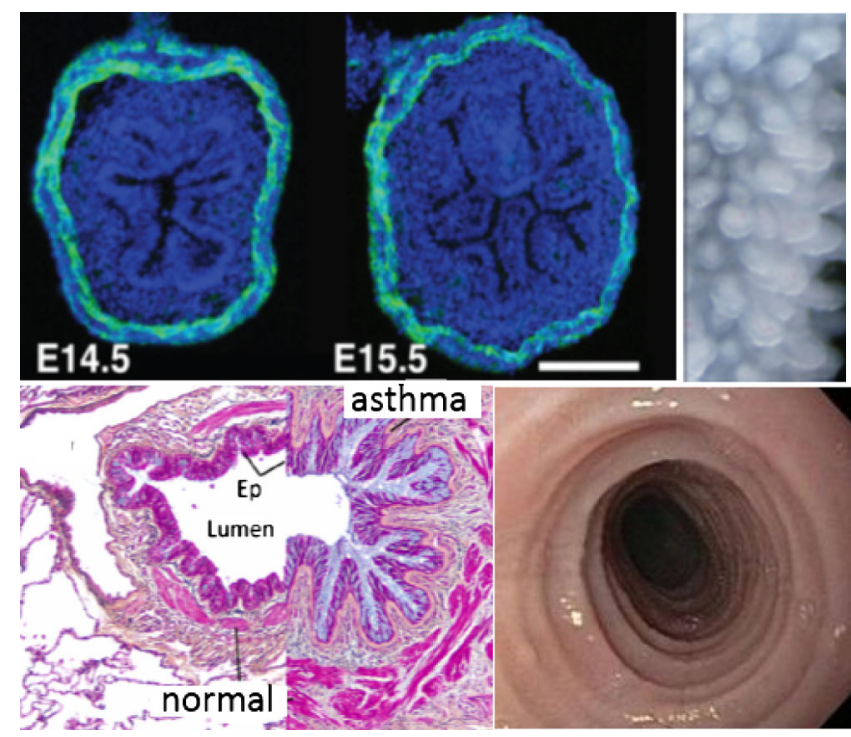

FIG. 1 (color online). Top: circumferential folding (left) and intestinal villi (right) in developing mouse intestine (adapted from Ref. [5]). Bottom: circular folding in normal and asthmatic airway (left, adapted from Ref. [7]); longitudinal folding in eosinophilic esophagitis (right, adapted from Ref. [8]). 
conditions are often characterized by the disruption of the normal homeostatic tension, which may trigger tissue remodeling: the epithelial layer starts to thicken and abnormal mucosal patterns arise, for example during asthma in the airways [7] and eosinophilic esophagitis in the esophagus [9] (see Fig. 1, bottom).

Recent research efforts focus on understanding the role of elasticity in generating a particular shape [10] with many applications in living systems including tumors $[11,12]$, the brain [13], skin [14], flowers [15], and trees [16]. Early attempts to explain emerging epithelial patterns in tubular tissues have considered simplified models with either a compressed thin sheet [17-20] or a growing flat film on a soft elastic substrate $[21,22]$. Further work has used finite elasticity to study the circumferential folding of growing tubular tissue using both linear stability analysis [23,24] and numerical methods $[25,26]$. Yet, even recent numerical simulations model the outer boundary as rigidly confined [5] and fail to realistically reproduce the physiology of a stress-free growing outer layer. Moreover, despite intense analytical and numerical efforts, the role of geometrical and mechanical factors in biological pattern formation has not been quantified, being still far from completely understood.

This work aims at studying the emergence and the nonlinear dynamics of epithelial patterns in a bilayered soft tube, which is allowed to grow freely in the absence of spatial confinements. Our scope is to provide quantitative predictors for differential growth between the layers and explore how their geometry and elastic properties regulate surface morphology.

We model the tubular tissue as a nonlinear elastic material with an inner layer, the endoderm, and an outer layer, the mesoderm, and distinguish their physical fields by the subscripts en and me. Since soft tissues are mainly composed of water, we assume that both layers are incompressible [27]. We adopt cylindrical coordinate systems and let $\mathbf{X}(R, \Theta, Z)$ and $\mathbf{x}(r, \theta, z)$ be the material and spatial position vectors in the ungrown reference and grown final configurations $\mathcal{B}_{0}$ and $\mathcal{B}_{a}$. The initial tubular tissue has an inner and outer radius of $R_{i}$ and $R_{o}$, respectively, while $R_{m}$ indicates the radius at the endodermmesoderm interface. We introduce the geometric aspect ratios $H_{\mathrm{en}}=R_{m} / R_{i}$ and $H_{\mathrm{me}}=R_{o} / R_{m}$ and denote the fixed axial length as $L$ (see Fig. 2). To model volumetric growth, we use the classical multiplicative decomposition of the deformation gradient $\mathbf{F}=\partial \mathbf{x} / \partial \mathbf{X}=\mathbf{F}_{e} \mathbf{F}_{g}$ into an elastic tensor $\mathbf{F}_{e}$ and a growth tensor $\mathbf{F}_{g}$ [28]. We assume that both layers grow homogeneously and isotropically as $\mathbf{F}_{g}^{(k)}=g_{k} \mathbf{I}$ with $k=\{$ en, me $\}$, where $\mathbf{I}$ is the unit tensor and $g_{\mathrm{en}}$ and $g_{\mathrm{me}}$ are the growth multipliers of the endoderm and mesoderm. We model both layers as incompressible neo-Hookean with elastic energy densities

$$
W_{k}=\mu_{k}\left[\left(F_{e}^{k}\right)_{i i}^{2}-3\right]-p_{k}\left[\operatorname{det}\left(\mathbf{F}_{e}^{k}\right)-1\right],
$$

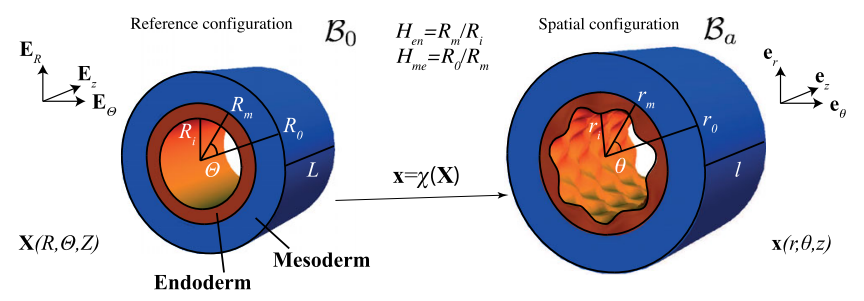

FIG. 2 (color online). Geometry of two-layered tubular tissue with inner endoderm and outer mesoderm in ungrown reference configuration $\mathcal{B}_{0}$ and grown residually stressed configuration $\mathcal{B}_{a}$.

where $\mu_{k}$ are the shear moduli, $p_{k}$ are the Lagrange multipliers to enforce the local incompressibility constraint, and the dummy index $i$ implies the summation rule. The elastic tensors of the basic axisymmetric solution are diagonal, $\mathbf{F}_{e}^{k}=\operatorname{diag}\left(\lambda_{r}^{k}, \lambda_{\theta}^{k}, \lambda_{z}^{k}\right)$, with the principal stretches $\lambda_{z}^{k}=1 / g_{k}$, $\lambda_{\theta}^{k}=r_{k} /\left(g_{k} R_{k}\right)$, and $\lambda_{r}^{k}=\left(\lambda_{\theta}^{k} \lambda_{z}^{k}\right)^{-1}$. Incompressibility imposes that $r_{k}=\sqrt{g_{k}^{3} R_{k}^{2}+a_{k}}$ with $a_{\mathrm{en}}=\left(r_{i}^{2}-g_{\mathrm{en}}^{3} R_{i}^{2}\right)$ and $a_{\mathrm{me}}=\left(r_{m}^{2}-g_{\mathrm{me}}^{3} R_{m}^{2}\right)$. With the elastic energy densities in Eq. (1), the components of the Cauchy stress tensors $\operatorname{read}\left(\sigma_{k}\right)_{i j}=\left[\mu_{k}\left(\lambda_{k}\right)_{i i}^{2}-p_{k}\right] \delta_{i j}$, where $\delta_{i j}$ is the Kronecker delta and the indices $\{i, j\}$ span over the cylindrical coordinates $\{r, \theta, z\}$. Evaluating the equilibrium condition, $\operatorname{div}\left(\boldsymbol{\sigma}_{k}\right)=\mathbf{0}$, in the radial direction yields the following equations

$$
\begin{aligned}
\left(\sigma_{\mathrm{en}}\right)_{r r} & =\mu_{\mathrm{en}} \int_{r i}^{r} \frac{1}{r}\left[\left(\lambda_{\mathrm{en}}\right)_{r}^{2}-\left(\lambda_{\mathrm{en}}\right)_{\theta}^{2}\right] d r \\
\left(\sigma_{\mathrm{me}}\right)_{r r} & =-\mu_{\mathrm{me}} \int_{r}^{r o} \frac{1}{r}\left[\left(\lambda_{\mathrm{me}}\right)_{r}^{2}-\left(\lambda_{\mathrm{me}}\right)_{\theta}^{2}\right] d r .
\end{aligned}
$$

At the inner and outer boundaries, the stresses satisfy the stress-free boundary condition $\left(\sigma_{\mathrm{en}}\right)_{r r}\left(r_{i}\right)=0$ and $\left(\sigma_{\mathrm{me}}\right)_{r r}\left(r_{o}\right)=0$. At the interface, the stresses are continuous, $\left(\sigma_{\mathrm{en}}\right)_{r r}\left(r_{m}\right)=\left(\sigma_{\mathrm{me}}\right)_{r r}\left(r_{m}\right)$. Solving the resulting set of equations for the interface coordinate $r_{m}$ provides the basic axisymmetric solution of the elastic problem.

To study the onset of endodermal pattern formation, we perform a linear stability analysis on the basic axisymmetric solution. Using the theory of incremental displacements superposed on finite deformations [29], we perturb the basic solution with an infinitesimal perturbation $\delta \mathbf{x}_{k}(r, \theta, z)=\left[u_{k}, v_{k}, w_{k}\right]^{T}$. For quasistatic growth, the first-order expansions of the elastic tensors become $\overline{\mathbf{F}}_{e}^{k}=\left[\mathbf{I}+\delta \mathbf{F}^{k}\right] \mathbf{F}_{e}^{k}$, where $\delta \mathbf{F}^{k}=\partial\left(\delta \mathbf{x}_{k}\right) / \partial \mathbf{x}$ are the gradients of the incremental deformation and barred quantities indicate perturbed fields. With $\overline{\boldsymbol{\sigma}}_{k}=\boldsymbol{\sigma}_{k}+\delta \boldsymbol{\sigma}_{k}$, the incremental Cauchy stress components follow from differentiating Eq. (1) as

$$
\left(\delta \sigma_{k}\right)_{i j}=L_{i j l m}^{k} \delta F_{m l}^{k}+p_{k} \delta F_{i j}^{k}-\delta p_{k} \delta_{i j} .
$$

Here $\delta p$ are the incremental Lagrange multipliers and $L_{i j l m}^{k}=\mu_{k} \delta_{j m} \delta_{i l}\left(\lambda_{i}^{k}\right)^{2}$ are the instantaneous elastic moduli. 

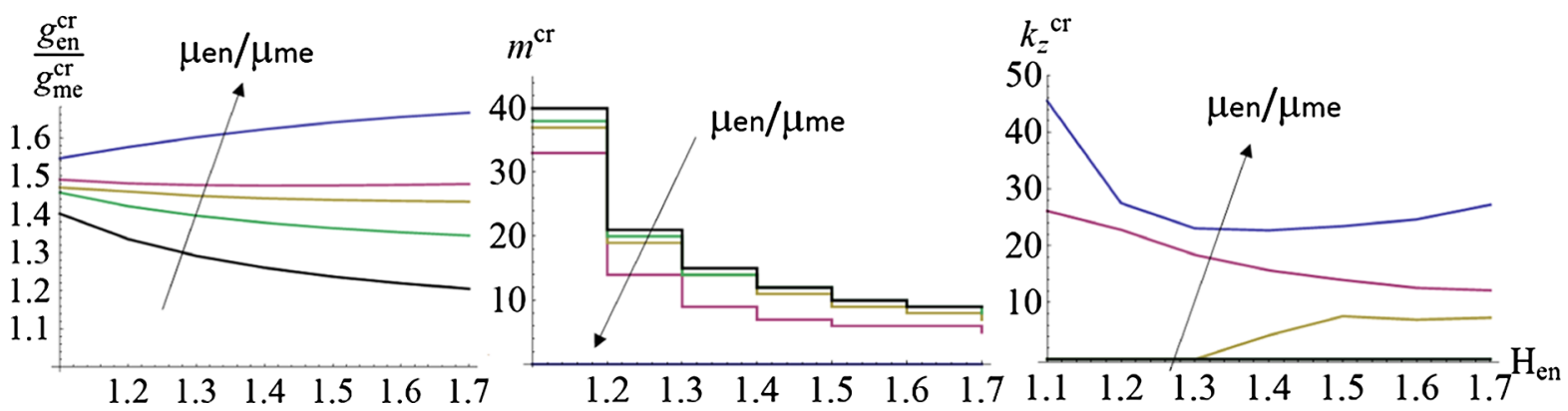

FIG. 3 (color online). Critical differential growth ratios $g_{\mathrm{en}}^{\mathrm{cr}} / g_{\mathrm{me}}^{\mathrm{cr}}$ (left), circumferential mode $m^{\mathrm{cr}}$ (center), and longitudinal mode $k_{z}^{\mathrm{cr}}$ (right) for varying aspect ratios of the inner layer $H_{\mathrm{en}}$ at fixed $H_{\mathrm{me}}=1.8$. Marginal stability curves are shown for elastic ratios $\mu_{\mathrm{en}} / \mu_{\mathrm{me}}=\{1 / 3,1 / 5,1 / 5.3,1 / 6,1 / 10\}$. For the sake of clarity we depict $k_{z}$ as a continuous parameter, assuming $L \gg R_{o}$.

We solve the incremental equilibrium equation $\operatorname{div}\left(\delta \boldsymbol{\sigma}_{k}\right)=\mathbf{0}$, together with the incremental incompressibility condition

$$
\left(u_{k}\right)_{, r}+\frac{u_{k}+\left(v_{k}\right)_{, \theta}}{r}+\left(w_{k}\right)_{, z}=0,
$$

and the stress-free incremental boundary conditions $\left(\delta \sigma_{\mathrm{en}}\right)_{r j}\left(r_{i}\right)=0$ and $\left(\delta \sigma_{\mathrm{me}}\right)_{r j}\left(r_{o}\right)=0$ for $j=\{r, \theta, z\}$. To solve the incremental problem, we assume a variable separation in the incremental fields and search for a sinusoidal solution:

$$
\begin{aligned}
\left\{u_{k}, \delta p_{k}\right\} & =\left\{U_{k}(r), Q_{k}(r)\right\} \cos (m \theta) \cos \left(k_{z} z\right) \\
v_{k} & =V_{k}(r) \sin (m \theta) \cos \left(k_{z} z\right) \\
w_{k} & =W_{k}(r) \cos (m \theta) \sin \left(k_{z} z\right)
\end{aligned}
$$

where $m$ and $k_{z}=2 \pi n / L$ (with $m, n \in \mathbb{N}$ ) are the circumferential and longitudinal modes, respectively, and $U_{k}(r)$, $V_{k}(r), W_{k}(r), Q_{k}(r)$ are four scalar functions. Thus, we can recast the incremental problem into a system of ordinary differential equations [30], and we define a robust method to get the numerical solutions [31]. Figure 3 illustrates the resulting marginal stability curves for the critical growth multipliers $g_{\mathrm{en}}^{\mathrm{cr}} / g_{\mathrm{me}}^{\mathrm{cr}}$ and the critical wave numbers $m^{\mathrm{cr}}$ and $k_{z}^{\text {cr }}$ for varying geometric aspect ratios $H_{\mathrm{en}} / H_{\mathrm{me}}$ and varying elastic aspect ratios $\mu_{\mathrm{en}} / \mu_{\mathrm{me}}$. The elastic ratio $\mu_{\mathrm{en}} / \mu_{\mathrm{me}}$ turns out to be a key regulator of epithelial pattern selection: large (small) values of $\mu_{\mathrm{en}} / \mu_{\mathrm{me}}$ favor the emergence of longitudinal (circumferential) wrinkling patterns, while both patterns emerge simultaneously for elastic parameters of the same magnitude. The geometrical ratio $H_{\mathrm{en}} / H_{\mathrm{me}}$ has a similar effect. This suggests that a competition between the bulk elastic energies is a major driver for pattern selection in tubular tissues.

To explore the nonlinear dynamics of pattern selection, we perform numerical simulations using the finite element program ABAQUS/STANDARD. We discretize the nonlinear problem of finite growth using trilinear hybrid brick elements C3D8H for both layers with 800 elements in the circumferential direction, 12 elements in the radial direction, and 100 elements in the longitudinal direction. We perform simulations for varying geometric ratios $H_{\text {en }} / H_{\text {me }}$ and elastic ratios $\mu_{\text {en }} / \mu_{\text {me }}$. We model volumetric growth as a pseudodynamic process similar to thermal dilatation and gradually increase the growth multiplier in time. To investigate the biologically relevant postbuckling behavior of the growing tissue, we introduce a small sinusoidal imperfection at the inner surface, with an amplitude of $\varepsilon=0.001 R_{o}$ and circumferential and axial modes $m^{\text {cr }}$ and $k_{z}^{\text {cr }}$ from the linear stability analysis. To validate the numerical solution, we confirm that the numerically calculated critical growth ratios $g_{\mathrm{en}}^{\mathrm{cr}} / g_{\mathrm{me}}^{\mathrm{cr}}$ are consistent with their theoretical predictions. We identify critical growth numerically at the time point where the total elastic energy first decreased more than $1 \%$ compared to the energy for the basic axisymmetric solution and find excellent agreement with the analytical solution.

Figure 4 summarizes the results in morphological phase diagrams of the emerging epithelial surface patterns. The numerical simulations display a wrinkle-to-fold transition in the postbuckling regime. The progressive increase of epithelial undulations eventually leads to surface selfcontact, an elastic singularity, which is often referred to as creasing [34]. As both the geometric ratio $H_{\mathrm{en}} / H_{\mathrm{me}}$ and the stiffness ratio $\mu_{\mathrm{en}} / \mu_{\mathrm{me}}$ decrease, the circumferential mode $m^{\text {cr }}$ increases while the axial wave number $k_{z}^{\text {cr }}$ decreases. This implies that circumferential (longitudinal) folding occurs when the elastic confinement of the outer mesoderm is strong (weak) enough: the total elastic energy, which includes both thickness and stiffness ratios, is the main morphological driver of the instability.

The reported shear moduli of embryonic gastrointestinal tissue range from $\mu_{\mathrm{en}}=120-700 \mathrm{~Pa}$ [5] to $\mu_{\mathrm{me}}=1 \mathrm{kPa}$ [35]. Our results are thus in excellent agreement with empirical observations: gastrointestinal embryonic tissues with a thicker outer layer, e.g., the esophagus with $H_{\mathrm{en}} / H_{\mathrm{me}} \simeq 1.8 / 1.9$ [36], fold circumferentially, while tissues with a thinner outer layer, e.g., the jejunum with $H_{\mathrm{en}} / H_{\mathrm{me}} \simeq 1.1 / 1.4$ [37], fold longitudinally like the valves of Kerckring. Our results are also in qualitative agreement with recent biological experiments on 

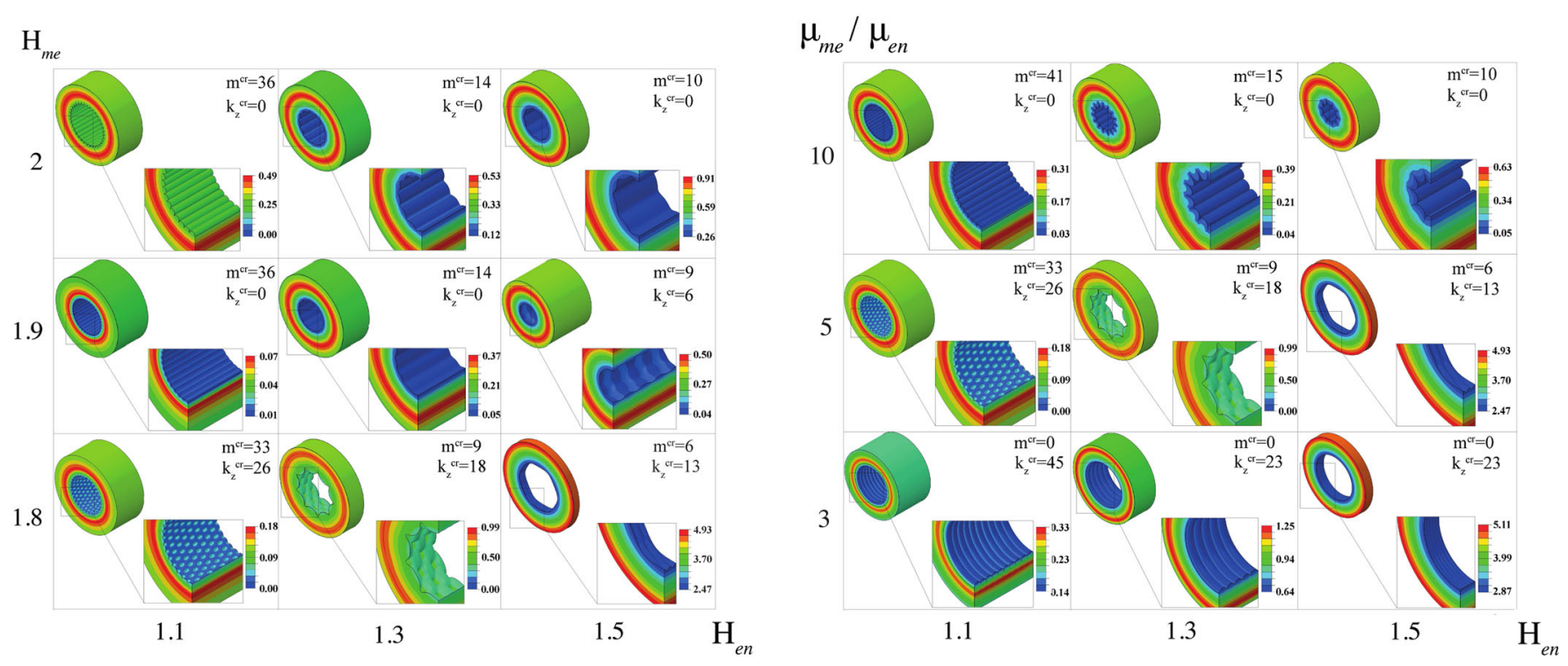

FIG. 4 (color online). Morphological phase diagrams of emerging epithelial surface patterns from numerical simulations in the postbuckling regime. The different surface morphologies are displayed for varying geometrical aspect ratios $H_{\text {en }}$ and $H_{\text {me }}$, at fixed $\mu_{\mathrm{en}} / \mu_{\mathrm{me}}=1 / 5$ (left) and for varying elastic ratios $\mu_{\mathrm{en}} / \mu_{\mathrm{me}}$ and geometries $H_{\mathrm{en}}$ at fixed $H_{\mathrm{me}}=1.8$ (right). The critical circumferential mode $m^{\text {cr }}$ and longitudinal wave number $k_{z}^{\text {cr }}$ correspond to the results of the linear stability analysis.

embryonic intestines: circumferential folding patterns emerge on the mucosal surface of chick embryos with $\mu_{\mathrm{en}} / \mu_{\mathrm{me}} \ll 1$ where the mesoderm is highly stiffened by early muscle differentiation, while square folding patterns appear in mouse embryos where both layers have similar stiffnesses of $\mu_{\mathrm{en}} / \mu_{\mathrm{me}} \simeq 1.5$ [5]. Further experiments on mouse embryonic intestines provide an estimate of the growth ratio at the onset of the instability with $g_{\mathrm{en}} / g_{\mathrm{me}}=$ 1.1 in the duodenum and 1.4 in the large intestine [38], both well within the expected range of our model predictions.

Our morphological phase diagrams can also provide a mechanistic interpretation for why the pathological thickening of the mucosal layer, e.g., during eosinophilic esophagitis, induces a transition from circumferential to longitudinal folding [9]. In intermediate regimes where the two elastic energies of the layers are of the same order both folding modes evolve simultaneously, giving rise to a square pattern, which eventually forms protruded fingers similar to the villi in the intestinal mucosa. Interestingly, if growth continues beyond the linear stability threshold, these surface patterns undergo a secondary bifurcation characterized by period doubling [39]. This induces a second transition, from square to hexagonal surface morphologies, characteristic for surface patters similar to the crypts of Lieberkühn in the large intestine. Ultimately, we aim to establish enhanced numerical tools to investigate these doubling patterns, since the onset of these secondary bifurcations often occurs at large growth multipliers, which are associated with extreme epithelial deformations.
In conclusion, we have investigated the pattern selection and nonlinear dynamics in the unconstrained growth of two-layered tubular tissues. Our linear stability analysis has provided quantitative estimates of the effects of geometry and elasticity on emerging surface morphologies. Our numerical simulation has demonstrated that after this initial bifurcation, the surface undergoes a wrinkle-to-fold transition in the nonlinear regime, forming a variety of characteristic epithelial structures found in gastrointestinal tissues. Understanding the interplay between geometry and elasticity during organogenesis provides valuable insight into how mechanical factors can regulate tissue growth and cell differentiation under both physiological and pathological conditions. This study highlights the need for further experimental investigation of these geometrical and mechanical cues at the early embryonic development. Ultimately, the presented results can be used to guide the microfabrication of functionalized surfaces with wide applications in various fields of tissue engineering.

We acknowledge partial funding from the France Stanford Center for Interdisciplinary Studies and Institut National de la Santé Et de la Recherche Médicale (INSERM) Grant No. OTPJ12U170. We thank M. Taffetani for technical assistance.

*ekuhl@stanford.edu

[1] I. R. Sanderson and W. A. Walker, Development of the Gastrointestinal Tract 1, PMPH-USA (2000).

[2] S. Yasugi, Dev. Growth Differ. 35, 1 (1993). 
[3] H. Gray, Anatomy of the Human Body (Lea \& Febiger, Philadelphia, 1918).

[4] T. K. Noah, B. Donahue, and N. F. Shroyer, Exp. Cell Res. 317, 2702 (2011).

[5] A. E. Shyer, T. Tallinen, N. L. Nerurkar, Z. Wei, E. S. Gil, D. L. Kaplan, C. J. Tabin, and L. Mahadevan, Science 342, 212 (2013).

[6] V. A. McLin, S. J. Henning, and M. Jamrich, Gastroenterology 136, 2074 (2009).

[7] J. C. Hogg et al., N. Engl. J. Med. 350, 2645 (2004).

[8] http://en.wikipedia.org/wiki/eosinophilic_esophagitis.

[9] A. Mishra et al., Gastroenterology 134, 204 (2008).

[10] A. Goriely and M. Ben Amar, Phys. Rev. Lett. 94, 198103 (2005).

[11] D. Ambrosi and F. Mollica, Int. J. Eng. Sci. 40, 1297 (2002).

[12] P. Ciarletta, Phys. Rev. Lett. 110, 158102 (2013).

[13] S. Budday, C. Raybaud, and E. Kuhl, Sci. Rep. 4, 5644 (2014).

[14] P. Ciarletta, M. Destrade, and A. L. Gower, Q. J. Mech. Appl. Math. 66, 273 (2013).

[15] H. Liang and L. Mahadevan, Proc. Natl. Acad. Sci. U.S.A. 106, 22049 (2009).

[16] L. Bertinetti, F. D. Fischer, and P. Fratzl, Phys. Rev. Lett. 111, 238001 (2013).

[17] L. Golubovic, D. Moldovan, and A. Peredera, Phys. Rev. E 61, 1703 (2000).

[18] D. Moldovan and L. Golubovic, Phys. Rev. E 60, 4377 (1999).

[19] D. Moldovan and L. Golubovic, Phys. Rev. Lett. 82, 2884 (1999).

[20] D. Drasdo, Phys. Rev. Lett. 84, 4244 (2000).

[21] E. Hannezo, J. Prost, and J.-F. Joanny, Phys. Rev. Lett. 107, 078104 (2011).

[22] M. Nelson, J. King, and O. Jensen, Math. Biosci. 246, 229 (2013).
[23] D. Moulton and A. Goriely, J. Mech. Phys. Solids 59, 525 (2011).

[24] P. Ciarletta and M. BenAmar, J. Mech. Phys. Solids 60, 525 (2012).

[25] B. Li, F. Jia, Y.-P. Cao, X.-Q. Feng, and H. Gao, Phys. Rev. Lett. 106, 234301 (2011).

[26] S. Budday, P. Steinmann, and E. Kuhl, J. Mech. Phys. Solids 72, 75 (2014).

[27] J. D. Humphrey and F. C.P. Yin, Biophys. J. 52, 563 (1987).

[28] E. K. Rodriguez, A. Hoger, and A. D. McCulloch, J. Biomech. 27, 455 (1994).

[29] R. W. Ogden, Non-Linear Elastic Deformations (Dover Publishers, New York, 1997).

[30] A. Stroh, J. Math. Phys. 41, 77 (1962).

[31] See Supplemental Material at http://link.aps.org/ supplemental/10.1103/PhysRevLett.113.248101 for further details on the theoretical derivation and the numerical methods, which include Refs. [30,32,33].

[32] S. Biryukov, Sov. Phys. Acoust. 31, 174 (1985).

[33] A. N. Norris and A. Shuvalov, Q. J. Mech. Appl. Math. 63, 401 (2010).

[34] Y. Cao and J. W. Hutchinson, Proc. R. Soc. A 468, 94 (2012).

[35] T. Savin, N. A. Kurpios, A. E. Shyer, P. Florescu, H. Liang, L. Mahadevan, and C. J. Tabin, Nature (London) 476, 57 (2005).

[36] D. Menard, Microsc. Res. Tech. 31, 215 (1995).

[37] M. A. Malas, R. Aslankoç, B. Üngör, O. Sulak, and Ö. Candir, Early human development 74, 109 (2003).

[38] R. Sbarbati, J. Anat. 135, 477 (1982).

[39] See Supplemental Material at http://link.aps.org/ supplemental/10.1103/PhysRevLett.113.248101 for movies S1 and S2 showing the secondary bifurcations with period doubling. 